\title{
Research on the Application of the Concept of Extreme Mode
}

\author{
Wei Liu* \\ School of Business \\ North China Institute of Science and Technology \\ Beijing, China \\ *Corresponding author \\ Jianyuan Gao \\ School of Business \\ North China Institute of Science and Technology \\ Beijing, China
}

\author{
Chenyu Liu* \\ School of Economics and Management \\ Chongqing University of Posts and Telecommunications \\ Chongqing, China \\ *Corresponding author \\ Xiaohong Chen \\ China mines safety report \\ North China Institute of Science and Technology \\ Beijing, China
}

\begin{abstract}
A new concept is often the result of practice requires. On the basis of the concept of modal, this paper puts forward the concept of Extreme Mode. The Extreme Mode not only contains the total number of units in the most sign values, also contains various units of the number of times many sign values, which may have several. The Extreme Mode is the special case of Extreme Mode, and the largest Extreme Mode. The Extreme Mode can intuitively show the objective phenomena in the allocation of central tendency in series and several minor central tendencies. The Extreme Mode is the statistics of the concept of modal perfect, which has extensive application value. For example, In the analysis of safety accident prevention, we should not only consider the most frequently occur accident of accident (mode), and analysis of all kinds of accidents. When arranging production specifications, enterprise should consider not only the largest sales product specifications, and should consider various specifications of the market. Therefore, the Extreme Mode application range than the Modal of the range.
\end{abstract}

Keywords-statistics; arithmetic mean; mode; maximizing modal

\section{INTRODUCTION}

Mode depends on the distribution of the number concentration condition. When times were significantly skewed distribution, which has more representative than other average index with it reflects the phenomenon. Due to the Mode only by how much the number distribution, which is not affected by heavy extreme big or small value, this has the high representative compared with arithmetic average. Likewise, the Mode has the following disadvantages: First, which is affected little by the variable value changes, lack of sensitivity; second, a series can appear more than one Mode, namely the phenomenon of the representative value is more than one, it is hard to compare and show that this average performance level is flawed; Third, in the frequency sequence, the small number of cases may not have the Mode, so you can not find out the Mode to calculate the average index[1].

This paper aimed at the above the second case to introduce the concept of the Extreme Mode, to solve practical problems Mode concepts too narrow in the practical problems.

\section{The CONCEPT OF MOdE AND Put FoRWARD OF THE CONCEPT OF EXTREME OF MODE}

\section{A. The Concept of Mode}

The Mode is a sign that the overall number of units in the most value, and is the symbol of the most common, most often in value. It can intuitively show the objective phenomena in the allocation of central tendency in series. It is the most general, most general overall variable values. Thus it can be used to explain social economic phenomenon of average [1]. Denoted: $\mathbf{M}_{0}$. Modal has wide application in practical work. For example, trade the price of a commodity on the market the most common level; Mass production of shoes, socks, hats and so on the most common size; Employees wages, the most common one of the most common age of the students, and so on, are often used to indicate the overall units number one sign value of average.

Mode characteristics: A set of data of variable value in the most times, more suitable for the data volume and central tendency obviously, which is not affected by extreme value (lack of sensitivity), a set of data may not have the number or several Mode. Based on the definition of the number, you can see that the number is only within the overall unit for several big enough to be meaningful, If overall unit number is less, although the frequency distribution is concentrated, so there seems little point in the number of the representative. If the overall unit number is more, but the number distribution of concentration, so and you can't have all the number is not the number. If more overall unit, and the overall mark in the most times value has two, it is double the number.

The method of solving Mode: the data grouping, coding sequence number distribution; through the observation to find number appear most that sign value. The number represented by the economic phenomenon of general level is not affected by extreme value, also is not affected by value assumption in the opening way. The special role and representing the general level of the Mode cannot substitute by arithmetic average and harmonic average. 


\section{B. The Concept of Extreme Mode}

A new concept of produce is often the result of practice requires. This paper argues that the concept of the Mode is too narrow, and not conducive to solve practical problems, this paper puts forward the concept of Extreme Mode. Extreme Mode is the value of statistical item compared with neighboring units' occurrences, is the overall units extremely common, often appear in the flag values.

It can intuitively show the objective phenomena in the distribution of sequence of number concentration trend. Visible, the Extreme Modes contain the most times and a sign of much value in the overall. It not only can intuitively show the objective phenomena in the allocation of central tendency in series and several central tendencies. Mode is a special Mode, and the number must be Extreme Mode, but the largest number is not necessarily the number.

\section{THE COMPARISON OF THE CONCEPT OF THE MODE AND THE EXTREME MODE}

\section{A. Un-grouped Data with the Mode and the Extreme Mode}

Example 1: the raw data: 25, 28, 28, 28, 28, 36, 42, 42, 42, 42, 50 and 52. The series has 2 Modes, namely 28 and 42; the series also has 2 Extreme Modes, namely 28 and 42. At this time, the Mode is the Extreme Mode.

Example 2: the original data: 25, 28, 28, 28, 28, 36, 42, 42, 42, 50 and 52. The sequence of the modal definition is only 28 , but there are two Extreme Modes, namely 28 and 42. Among these, Extreme Mode 28 is also the Mode.

Comparison Case 1 and case 2, according to the traditional concept of the Mode, in the case 2, mark value 42's times is 3 , a less than 28, which cannot as the Mode. According to the actual situation, sometimes the Extreme Mode has the big application value. For example, for enterprises, especially large enterprise, not only to find the Mode, so that production specifications conform to the public, also found enough customer requirements (part of the larger Extreme Mode). For small and medium-sized enterprises implement the strategy of "gap", from a statistical point of view is to find the Extreme Mode. There is the future for the small and medium-sized enterprises to find those niche market as a target market and profits which the big companies have not found, or doesn't want to do big business..

\section{B. Monomial Series with Mode and Extreme Mod}

Example 3: there are 200 workers in workshop at a factory, March and April processing on the number of parts as shown in table 1. In March, according to the definition of the Extreme Mode, there are two Mode, namely M03 = 34, 35 (points), the processing Mode in April M04 = 35 (points); And according to the concept of Extreme Mode, march and April of the number of parts processing Extreme Modes are the same, namely, 32, 33, 34, 35.
TABLE I. A WORKSHOP IN MARCH PROCESSING PLANT PARTS NUMBER STATICAL TABLE

\begin{tabular}{|c|c|c|c|c|}
\hline \multirow{2}{*}{$\begin{array}{c}\text { Number of } \\
\text { parts } \\
\text { (points) }\end{array}$} & \multicolumn{2}{|c|}{$\begin{array}{c}\text { The number of parts } \\
\text { processed in March }\end{array}$} & \multicolumn{2}{c|}{$\begin{array}{c}\text { Number of parts } \\
\text { processed in April }\end{array}$} \\
\cline { 2 - 5 } & $\begin{array}{c}\text { Number of } \\
\text { workers } \\
\text { (Individual) }\end{array}$ & $\begin{array}{c}\text { Proportion } \\
\text { (\%) }\end{array}$ & $\begin{array}{c}\text { Number of } \\
\text { workers } \\
\text { (Individual) }\end{array}$ & $\begin{array}{c}\text { Proportion } \\
\text { (\%) }\end{array}$ \\
\hline 32 & 24 & 12.0 & 24 & 12.0 \\
\hline 33 & 44 & 22.0 & 44 & 22.0 \\
\hline 34 & 66 & 33.0 & 65 & 32.5 \\
\hline 35 & 66 & 33.0 & 67 & 33.5 \\
\hline total & 200 & 100.0 & 200 & 100.0 \\
\hline
\end{tabular}

IV. CAses Show That The APPlication of the CONCEPT OF THE MODE

\section{A. The Concept of Mode is Indispensable by Examples}

Example 4, a shoe factory to understand consumer needs which most want what type of shoes, a survey of a department store one sales, as shown in table 5 . Can be seen from table 5, $25.5 \mathrm{~cm}$ at most shoe sales, if we calculate arithmetic average which is $26.5 \mathrm{~cm}$, and this number is clearly no practical significance, and which is likely to mislead the producers, and directly use $25.5 \mathrm{~cm}$ shoes as our customers required size of the central tendency is not only convenient practical.

However, if the above problem becomes: the shoe factory how to arrange their own production of various specifications of the production shoes, it not only need to know the Mode and proportion, also need to know Extreme Mode and its proportion, namely the need to define the Extreme Mode and its proportion. As above case, they should not only know the Mode is $25.5 \mathrm{~cm}$, to make more production $25.5 \mathrm{~cm}$ shoes' decision-making, but also know the specific proportion of the Mode group (41.62\%), and also need to know the Extreme Modes (24.0, 24.5, 25.0, 26.0, 26.5, 27.0) and its proportion (4.92, 6.62, 9.85, 25.00, $8.00,4.00)$, such as enterprise according to the market to make plans for future production of 100000 pairs of shoes a year, should be how to arrange all kinds of shoe production of specific plans, as shown in table 2.

Calculation steps: first, look for the sales of the department store one quarter of the Extreme Modes, there are seven Extreme Modes, namely, 24.0, 24.5, 25.0, 25.5, 26.0, 25.5, 26.0, and 25.5 is the Mode, means that these sizes should be produced; Secondly calculate the proportion of the Extreme Modes; Finally, 100000 pairs of shoes production multiplied by the proportion of the Extreme Mode, the calculation results are the last column shown in table 2, these are the developer of next year a total of 100000 pairs of shoes in the Extreme Mode of production.

In table 1 for the above example 3, the parts are manufactured in April the number of 34 only one less than the number of production of 35, 34 is not Mode in April. In reality, companies sometimes focus the Mode, such as number of 34 parts in April, such as corporate training under the condition of limited resources, which should choose to produce parts of 34 groups as a key training, and should not choose production parts of 32 groups, because the number of group production parts 34 to 65 , more than 24 to 32 the number of production. 
Overall training, increase an average production, the output increases more.

TABLE II. TABLE THE SALES OF A DEPARTMENT STORE A

QUARTER STASTISTICS AND IN THE NEST YEAR PIAN PRODUCTION

\begin{tabular}{|c|c|c|c|c|}
\hline \multirow{2}{*}{$\begin{array}{c}\text { sequence } \\
\text { number }\end{array}$} & $\begin{array}{c}\text { Shoes no. } \\
\mathbf{( c m )}\end{array}$ & $\begin{array}{c}\text { Sales } \\
(\text { double) }\end{array}$ & $\begin{array}{c}\text { Proportion } \\
\mathbf{( \% )}\end{array}$ & $\begin{array}{c}\text { arrange production at } \\
\text { the levels of 100000 pairs } \\
\text { of shoes }\end{array}$ \\
\cline { 2 - 5 } & $(\mathbf{1})$ & $(\mathbf{2})$ & $(3)$ & $(4)$ \\
\hline 1 & 24.0 & 64 & 4.92 & 4923 \\
\hline 2 & 24.5 & 86 & 6.62 & 6615 \\
\hline 3 & 25.0 & 128 & 9.85 & 9846 \\
\hline 4 & 25.5 & 541 & 41.62 & 41615 \\
\hline 5 & 26.0 & 325 & 25.00 & 25000 \\
\hline 6 & 26.5 & 104 & 8.00 & 8000 \\
\hline 7 & 27.0 & 52 & 4.00 & 4000 \\
\hline & total & 1300 & 100.00 & 100000 \\
\hline
\end{tabular}

In addition, a company not only attaches importance to appear most frequently type of accident (accident Mode), also attaches great importance to the occurrences of most types(accident Extreme Modes), etc.

\section{CONCLUSIONS}

Extreme Mode is the value of statistical item compared with neighboring units' occurrences, is the overall units extremely common, often appear in the flag values (very common, very popular), which may have several. The Extreme Mode not only contains the overall number of units in the most value (the most common, the most popular), also includes a sign of the number of units appear once more (generally, popular). Extreme Mode can intuitively show the objective phenomena in the allocation of central tendency in series and several central tendencies.

The concept of Extreme Mode is perfect for the concept of Mode. Mode is a special case of the Extreme Mode, Mode is the biggest Extreme Mode, and the Mode must be the Extreme Mode, but the Extreme Mode is not necessarily the Mode. In practice, people should not only consider the overall number of units in the most value, and also need to study various units of the number of times many flag values, we need to research Extreme Mode, Highly visible Extreme Mode application scope is wide than modal.

\section{ACKNOWLEDGMENT}

This work was financially supported by Enterprise management project of North China Institute of Science and Technology (HBXJZD201403).

\section{REFERENCES}

[1] Wei Liu, Siyou Lei. Statistics [M], China mining university press, 2012, 1. 